\title{
Coherence a measure of the brain networks: past and present
}

\author{
Susan M. Bowyer
}

\author{
Correspondence: \\ Sbowyer1@hfhs.org \\ Department of Neurology, Henry \\ Ford Hospital, 2799 West Grand \\ Blvd, Detroit, Ml 48202, USA
}

\begin{abstract}
Brian connectivity describes the networks of functional and anatomical connections across the brain. The functional network communications across the brain networks dependent on neuronal oscillations. Detection of the synchronous activation of neurons can be used to determine the wellbeing or integrity of the functional connectivity in the human brain networks. Well-connected highly synchronous functional activity can be measured by Electroencephalography (EEG) or Magnetoencephalography (MEG) and then analyzed with several types of mathematical algorithms. Coherence is one mathematical method that can be used to determine if two or more sensors, or brain regions, have similar neuronal oscillatory activity with each other. Since the 1960's, coherence has generally been assessed on the similarity of the frequency content across EEG sensors. Recently coherence, after it has been imaged in the brain, has been used to assess how coherent or connected specific locations in the brain are networked together in several different neurological disorders. Statistical analysis can then be performed on the coherence results to verify evidence of normal or abnormal network activity in a patient. In this review we highlight how functional brain connectivity is assessed in Source space using coherence technique measured by MEG.
\end{abstract}

Keywords: Neuronal oscillations, Connectivity, Networks, Coherence, EEG, MEG

\section{() Biomed Central}

\section{Background}

The human brain is a vast network of connected pathways that communicate through synchronized electric brain activity along fiber tracts. The synchronized activity within this neuronal network can be detected by MEG and EEG then imaged using network connectivity analysis. Connectivity analyses of the brain are performed to map out the communication networks needed for the brain to function. A large number of neuroimaging brain studies in the past have found that there are specific regions in the brain that are specialized for processing certain types of information. Over the past 10 years advances in brain imaging techniques have revealed that these regions are connected and communicate with other specialized regions across networks in the brain. Sometimes these activations are linear and yet at other times these activations can be simultaneous. Neurological disorders and tumors can disrupt brain functions. Treatments and remediation have also been shown to change how the brain functions. This illustrates a more complex picture of the brain as a dynamic interconnected network, capable of plasticity and adaptation $[7,41]$.

(c) 2016 Bowyer. Open Access This article is distributed under the terms of the Creative Commons Attribution 4.0 International License (http://creativecommons.org/licenses/by/4.0/), which permits unrestricted use, distribution, and reproduction in any medium, provided you give appropriate credit to the original author(s) and the source, provide a link to the Creative Commons license, and indicate if changes were made. The Creative Commons Public Domain Dedication waiver (http://creativecommons.org/ publicdomain/zero/1.0/) applies to the data made available in this article, unless otherwise stated. 
Neuroimaging connectivity techniques for quantifying the brain networks use signal processing techniques that have been around for many decades [8]. There are three connectivity network types that are used to investigate communication within and across the brain. These network connection types are categorized as Structural, Functional and Effective $[15,19,40]$. Within these categories several different imaging hardware equipment and software programs are used to detect, measure and quantify the integrity of the network. Structural connectivity is based on detection of the fiber tracts that physically connect the regions of the brain. These are the anatomical network maps that indicate possible pathways that the signals can travel on in the brain [32, 49]. Functional connectivity identifies activity brain regions that have similar frequency, phase and/or amplitude of correlated activity. These areas may be involved in the resting state (i.e. task independent) or higher order information processing (i.e. task dependent) that is required for sensory responses, motor responses and intellectual or emotional processing. [45]. Effective connectivity uses the functional connectivity information and goes one step further and determines the direct or indirect influence that one neural system may have over another, more specifically the direction of the dynamic information flow in the brain $[8,28]$.

Effective and Functional Connectivity measurements can be analyzed in the Frequency Domain with methods such as Coherence and Phase synchrony or in the Time Domain with methods such as Correlation and Granger Causality. Coherence and Phase synchrony are common mathematical methods for quantifying frequency and phase dependent correlations of brain activity measured by two or more brain sensors. Functional connectivity does not determine the specific direction of information flow in the brain. It just shows that these regions have similar signal content and therefor are most likely connected. This type of connectivity analysis is based on brain signals recorded by Electroencephalography (EEG), Magnetoencephalography (MEG), Functional Magnetic Resonance imaging (fMRI) and Positron Emission Tomography (PET). Effective connectivity determines which of the brain location are sending and/or receiving information this can be calculated using mathematical techniques such as Granger causality, Hilbert transform, transfer entropy and correlation [22]. Brain signal information for effective connectivity can best be found in brain activity recorded by EEG or MEG as these techniques have high temporal resolution to resolve the dynamic flow of information.

Brain networks are made up of populations of neurons that function in unison to send signals to other parts of the brain. There are several properties of the neuron that play an important role in generating membrane potential oscillations that can be detected by neuroimaging devices. Neurons communicate with other neurons by releasing one of over 50 different types of neurotransmitters in the brain, some of which are excitatory (stimulate the brain) and some are inhibitory (calm the brain) [9]. Voltagegated ion channels generate action potentials and periodic spiking membrane potentials which produces oscillatory activity and facilitates synchronous activity in neighboring neurons [34, 35]. Coherent neuronal communications are based on neurotransmission dynamics dictated by major neurotransmitters like the amino acids glutamate and GABA. Other important neurotransmitters include acetylcholine, dopamine, adrenaline, histamine, serotonin and melatonin [24, 44, 48]. These action potentials activate a neighboring population of neurons which in turn may affect other populations of neurons at a 
distance creating a network of connectivity. Coherence across a network is when these populations of neurons are active at the same time or in a time-related fashion with other populations, not a relationship of activity between individual neurons.

Synchronized activity of a significantly large population of neurons can give rise to large electric field oscillations with a concomitant magnetic field, which can be detected by EEG or MEG respectively [25]. MEG measures the ionic current flow inside of the neuron (primary currents) while EEG measure the return or volume currents outside of the neuron (secondary currents). Functional and effective connectivity techniques are dependent on calculating the communication of active neural signals that are oscillating over short and long periods of time. MEG and EEG techniques, with their excellent temporal resolution, are optimal for calculating connectivity. For a review of mathematical equations of connectivity measures used in EEG and MEG for neurologic disorders see $[19,40]$. PET and FMRI measure the secondary or metabolic response from neuronal activation [38]. These are both indirect measures of neuronal activation with low temporal resolution on the order of seconds. Since they lack the temporal resolution these two techniques cannot assess the electrophysiological basis for functional or effective connectivity.

Coherence is a mathematical technique that quantifies the frequency and amplitude of the synchronicity of neuronal patterns of oscillating brain activity. This technique quantifies the neuronal patterns of synchronicity measured between spatially separated scalp electrodes (Electroencephalogram) or coils (Magnetoencephalogram) [14]. Coherence is an estimate of the consistency of relative amplitude and phase between signals detected in coils or electrodes within a set frequency band. In sensor space if signals are in phase then their amplitudes will add, if they are out of phase the signals will subtract possibly reducing the coherence value. In source space the amplitude of the underlying source can be used to determine the strength of the connectivity. See Table 1 for the comparison of coherence characteristics in sensor space and source space. The method for quantifying the oscillations is to first apply a time-frequency decomposition technique such as the Fast Fourier transform (FFT), on a contiguous or slightly overlapping sequence of short data segments. This generates a sequence of amplitude/phase components for each narrow frequency bin (i.e. 2-4 Hz) of the FFT that spans the frequency (i.e. 1-100 Hz) content of the data. After transformation to a time frequency representation, the strength of network interactions can be estimated by calculation of coherence, which measures the synchrony between signals from different electrodes or

Table 1 Comparison of coherence characteristics in sensor space and source space

\begin{tabular}{lll}
\hline Characteristics & Coherence in Sensor space & Coherence in Source Space \\
\hline $\begin{array}{l}\text { Frequency components } \\
\text { Inverse solution }\end{array}$ & Yes & Yes \\
Localization & $\begin{array}{l}\text { No } \\
\text { Inferred by indicating } \\
\text { which electrode is active } \\
\text { and drawing lines }\end{array}$ & $\begin{array}{l}\text { Yes } \\
\text { is active and indicated by colored pixels }\end{array}$ \\
Raw MEG/EEG recordings & Yes & No, Data is processed first \\
Field spread effects on results & $\begin{array}{l}\text { Yes, the current flowing } \\
\text { across the scalp is detected } \\
\text { by many sensors simultaneously, } \\
\text { which leads to uncertainty in the } \\
\text { location of the brain activity. }\end{array}$ & $\begin{array}{l}\text { No, since the data is first imaged into the } \\
\text { brain. Then the coherence is calculated } \\
\text { provides more accurate information on } \\
\text { which brain regions are actual coherent. }\end{array}$ \\
\hline
\end{tabular}


coils at each FFT frequency bin. Coherence is a linear math method in the frequency domain for calculating neuronal networks. The result is a symmetrical matrix that provides no information on directionality. Coherence is the most common measure used to determine if different areas of the brain are generating signals that are significantly correlated (coherent) or not significantly correlated (not coherent). Strictly speaking coherence is a statistic that is used to determine the relationship between two data sets (i.e. coils or electrodes). It is used to determine if the signal content of two inputs are the same or different. If the signals measured by two electrodes or coils are identical then they have a coherence value of 1 ; depending on how dissimilar they are the coherent value will approach 0 .

Phase synchrony is another math signal processing technique that estimates the synchrony of oscillations in EEG and MEG data. This is the process where two or more cyclic signals tend to have oscillator activity that are the same (in phase) or out of synchrony (out of phase) by a relative phase angle. Phase synchrony measures how stable the phase difference (small or large) varies over a short period of time. Phase relationships can be examined by testing the stability of the signals phase differences across trials (phase-locking) over a single electrode or between pairs of electrodes [30]. This approach can yield estimates of the precision of local and long-range synchrony. Importantly, measures of phase-locking provide estimates of synchrony independent of the amplitude of oscillations. This is in contrast to measures of coherence where phase and amplitude are intertwined [46]. Phase synchrony is better used for short duration events such as in an evoked event. Phase is used to determine how much the two locations (recording sites) are interacting within a very narrow time window (milliseconds). A great analogy for understanding the difference between when to use coherence or phase synchrony analysis is Soldiers marching in a parade: phase synchrony is used to determine how synchronized their feet are marching in unison in a few steps, while coherence is used to see how synchronous their feet were marching in unison over the entire parade route.

Coherence and Phase synchrony are the building blocks for understanding brain connectivity, how the populations of neurons communicate around a network. Traditionally to determine if different areas of the brain are connected using EEG or MEG, a frequency analysis was performed to convert the original EEG or MEG data into its frequency content, then coherence analysis was used to obtain information about the temporal relationships of frequency components of populations of neurons at different recording sites (electrode or coil). The results of the coherence analysis were typically displayed in sensor space using a template of the head with lines connecting the electrodes or coils that are coherent with each other (called sensor space; Fig. 1) would represent the areas of the brain that were connected. The analysis of coherence and phase locking analysis between EEG electrode site [10, 17, 33, 37] and MEG sensors [16] (in sensor space) has been around for a long time.

Typically EEG Coherence in sensor space has been widely used in studying epileptiform activity to determine seizure onset zones. Brazier in 1972 [6] was the first to use coherence to detect the influence of one brain region over another during epileptic seizure. Later Gotman in 1981 [18] made the method more reliable by including more frequencies and validating the use of this method to detect inter hemispheric interactions. In the present day, Song et al. has shown that EEG coherence can be used to 


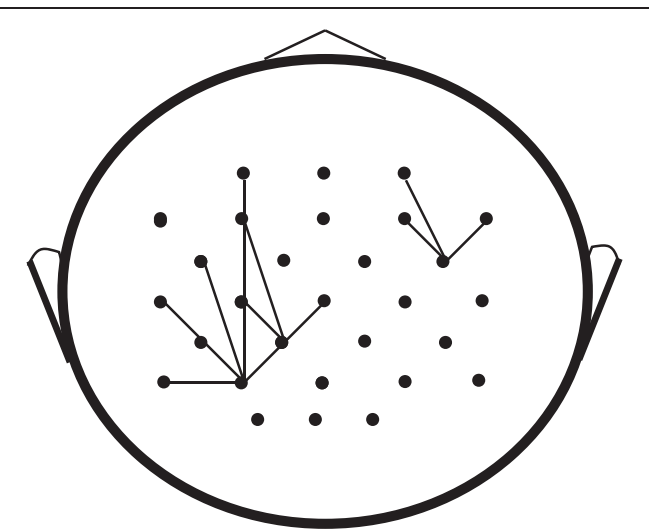

Fig. 1 An illustration of Sensor space coherence mapping. Black dots represent the locations of the recording EEG electrode (or MEG coil). The lines connecting 2 dots indicated there was high coherence between these two sensors

characterize a pattern of strong coherence centered on temporal lobe structures in several patients with epilepsy [43]. Coherence in sensor space has also been widely used to look at differences in patients with other brain disorders. Ozerdem et al. [39] found patients with a Bipolar disorder showed bilaterally diminished longdistance gamma coherence between frontal and temporal as well as between frontal and temporo-parietal regions compared with healthy controls. In patients with Alzheimer's the most common finding is a decrease in the alpha and beta band coherences between distant structures during resting state $[1,36]$. In the field of schizophrenia Yeragani et al. [50] found a decrease in sleep coherence in both beta and gamma frequency bands in first episode schizophrenia patients compared to normal controls. Uhlhaas et al. [47] Provided an in-depth review of abnormal neural oscillations and synchrony in this patient group. They review several studies that indicated that patients with schizophrenia have a reduced phase synchrony in the beta and gamma bands [47]. For a review of brain oscillations in several different brain disorders see [2].

One of the problems with this traditional way of mapping coherence in sensor space is the spread of the electric current over the surface of the head can be misleading. All electrodes or coils are the sum of the activity from several brain regions so it is hard to resolve the coherence to a specific brain location [42], since the coil or electrode will have electromagnetic signals from adjacent areas included in the sum of the signal.

Directionality of the network interaction cannot be determined from coherence alone and the exact amplitudes of the network interaction are not equal to region-to-region coherence amplitudes. Coherence does provide a global estimate of all important regions of network activity regardless of source amplitudes. Because of the need to minimize bias by increasing the number of data segments in calculations, coherence is not well suited for quantifying rapid temporal changes in synchronized activity. Rather it is best when used for long time series of data to identify sources of brain network activity that persist for long durations. Coherence analysis supplies information on the degree of synchrony of brain activity at different locations for each frequency, independent of power. However, individual time points with large amplitudes are more highly weighted in the FFT transform and subsequently in coherence calculations. This 
is in contrast to phase synchrony which utilizes instantaneous measurements of only the phase differences between signals.

In the past 10 years, developments in the computational analysis of the source localization for MEG have advanced the ability for connectivity to be imaged directly into the specific regions of the brain (called Source space; Fig. 2). This source space method provides better anatomical localization as well as the ability to perform group analysis, as the data can now be averaged across a standardized space. The high temporal resolution of MEG allows for investigations of function and effective connectivity with millisecond precision. It is possible now to study the mechanisms by which information is exchanged across brain regions, including oscillatory and synchronized neuronal activity. There have only been a handful of coherence studies performed in the past 10 years using different inverse methods (dipoles, minimum norm or beamformers) to perform source space coherence analysis across many different brain disorders. All of these diverse investigations show that coherence imaging in Source space provides a wealth of new information on the integrity and strength of the functional connectivity in a patient's brain networks.

In 2004 Hoechstetteret al. [28] originally introduced source coherence using dipoles. Brain electrical source analysis (BESA) was applied to create a discrete multiple source
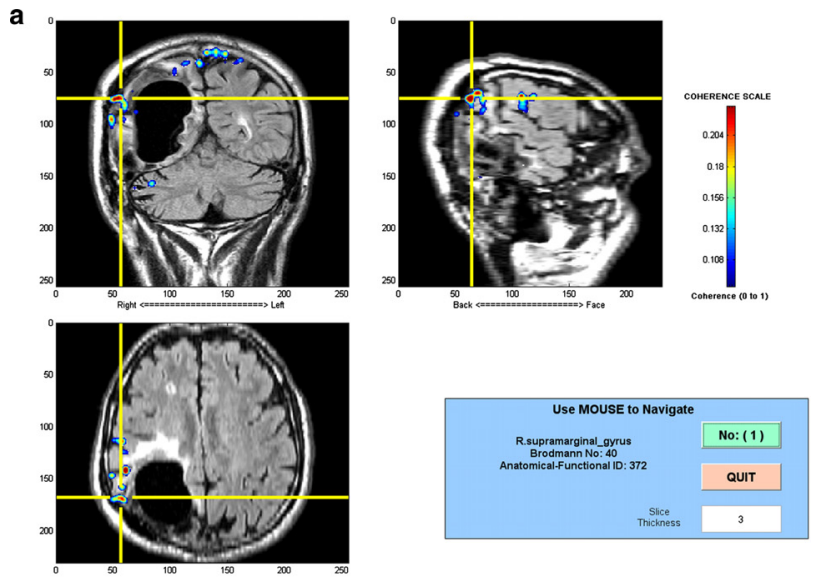

b

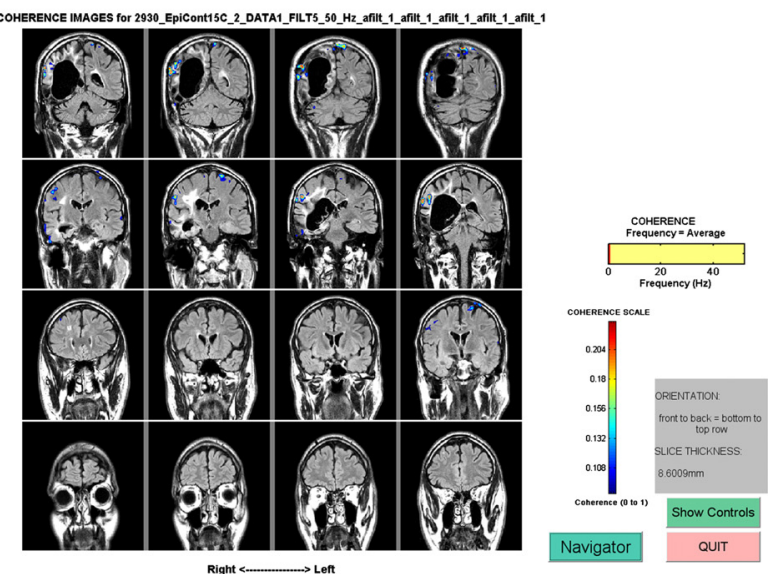

Fig. 2 An illustration of Source space coherence mapping. MEG- CSI analysis of the locations of coherent activity in the brain of a patient with epilepsy. Top 15 axial slices are the view of the entire brain. The 3 MRI slices below indicate the top coherent location of brain activity in the Right supra marginal gyrus 
model. This model used a source montage to transform the recorded data from sensor level into brain source space. The source waveforms are transformed into time-frequency space using complex demodulation. Magnitude-squared coherence between the brain sources revealed oscillatory coupling between sources. Since sensor spaces uses the MEG/ EEG waveforms this will include the signals from adjacent areas in the brain, but using source space a localizations is first performed using an inverse solutions which removes the current that may have spread in to the MEG or EEG coil/electrode, providing a specific regions that the coherent frequencies maybe coming from. This coherence method at the source level overcomes problems of localization and interpretation that are fundamental problems for coherence analysis at the sensor level [27].

In 2001 Gross et al. [20] used Dynamic imaging of coherent sources (DICS) to study neural interactions in the human brain. DICS uses a spatial filter to localize coherent brain regions and provides the time courses of their activity. Reference points for the computation of neural coupling may be based on brain areas of maximum power or other physiologically meaningful information, or they may be estimated starting from sensor coherences. The performance of DICS was evaluated with simulated data and illustrated with recordings of spontaneous activity in a healthy subject and a parkinsonian patient. Methods for estimating functional connectivity between brain areas can facilitate characterization of cortical networks involved in sensory, motor, or cognitive tasks and can help investigate pathological connectivity in neurological disorders. Gross et al. [21] found frontal, parietal and temporal beta coherence was relevant for the processing of stimuli in working memory.

Using MEG and a beamformer, Guggisberg et al. in 2007, investigated the functional connectivity in the alpha frequency in patients with brain lesions [23]. Neural activity in the brain was estimated using an adaptive spatial filtering technique where an index of functional connectivity was created using the mean imaginary coherence between brain voxels. In healthy subjects the somatosensory and language cortices had the highest alpha coherence. Patients with lesions had decreased alpha coherence that was diffuse and scattered across the brain. These patients also had decreased connectivity in the lesioned part of the brain compared with intact contralateral regions. In tumor patients without preoperative neurological deficits, brain areas showing decreased coherence could be surgically resected without the occurrence of postoperative deficits.

In 2007, Belardinelli et al. [3] investigated the cerebral networks involved in motor control in four young hemi-paretic patients with pre- and perinatally acquired brain lesions by means of MEG source coherence analysis. Previous TMS and fMRI studies on the same patients had investigated their residual ability to move the paretic hand by means of a reorganized primary motor cortex (M1) representation in the contralesional hemisphere. The purpose of this study was to identify the effects of a cerebral reorganization and the related dynamic aspects which allowed the patients to move their paretic arm. MEG signals were recorded simultaneously with EMG which was used a reference for coherence calculations. Coherence mapping in the beta frequency range $(14-30 \mathrm{~Hz})$ confirmed the relocation of motor functions from the lesioned (left) to the contralesional (right) hemisphere. Coupling direction analysis demonstrated that throughout pinch grip the coupling direction goes from M1 to cerebellum. This study verified the assumption that the intact hemisphere takes over motor control from the paretic (ipsilateral) hand in the presence of early unilateral brain lesion [3]. 
In 2010 dePasquale et al. [11] described MEG signatures of two well-characterized resting state networks: the dorsal attention and the default mode networks. Seed-based correlation mapping was performed using time-dependent MEG power reconstructed at each voxel within the brain. The analysis was performed on a 5 min resting state scan and was found to be similar to that observed with fMRI but confined to the same hemisphere as the seed region. Analyses taking into account the MEG activity is not stationary, showed transient formation of more complete resting state networks, including nodes in the contralateral hemisphere.

In 2011 Elisevich et al. showed that MEG Coherence source imaging (MEG-CSI) in source space can provide targets for successful surgical resections in patients with Epilepsy [12]. MEG-CSI used a current distribution technique, MR-FOCUSS to image the underlying sources based on a 10 min resting state scans. They found a positive predictive value for an Engel's class Ia outcome in $70 \%$ of the patients who had high coherence in the area of the later resection. More recently [13] looked at the regional and global functional connectivity of MEG coherence in patients with Epilepsy, based on a 1 min resting state scan. This study used a beamforming technique to image the underlying sources. They found that seizure freedom was seen in $87.5 \%$ of patients where increased connectivity was found in the region of the later resection.

Hinkley et al. used source space MEG to detect decreased and increased connectivity differences between patients with schizophrenia and control subjects, that may prove to be important target areas for treatment [26]. Two more recent MEG studies by [29, 5], have shown that neuronal patterns in the default mode network (DMN) during rest can be used to identify patients with schizophrenia compared to controls [29] used MEG to identify the DMN regions and found that the spatial distribution of DMN activity in the alpha band was similar to that found in previous fMRI studies [29]. The DMN and resting activities of the posterior cingulate cortex (PCC) were increased in schizophrenia patients, predominantly in the theta and alpha bands. They also showed that both the resting and DMN activities were augmented in the PCC in schizophrenia patients. Furthermore, schizophrenia patients exhibited decreased coherence between the PCC and medial pre frontal cortex (MPFC) in the gamma band at rest. A year later Bowyer et al. [5] used MEG-CSI, which had been applied successfully for detecting coherent areas in the cortical networks of patients with epilepsy [12], to investigate brain imaging Biomarkers of schizophrenia [5]. MEG-CSI analysis found increased regions of coherence across a large frequency range $(3-50 \mathrm{~Hz})$ in schizophrenia patients compared to controls in the medial and ventrolateral PFC and anterior cingulate cortex. These areas are involved in language, memory, decision making, empathy, executive and, higher cognitive functioning. They concluded that MEG-CSI can detect imaging biomarkers from resting state brain activity in schizophrenia patients that deviates from normal control subjects in several behaviorally salient brain regions. This technique can look at the strength and location of the connections that a specific activated location makes (Fig. 3). These techniques for imaging connectivity can be used to probe the pathophysiology of schizophrenia and possibly detect subtypes.

This same technique, MEG-CSI, has been used to look at the connectivity in patients with Panic disorder [4]; where they found coherence imaging values were significantly higher in panic patients compared to controls during a $10 \mathrm{~min}$, eyes open, resting state scan. They propose that additional research examining coherence values in Panic 


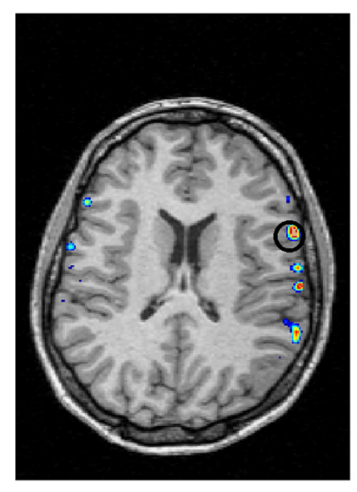

Right $\longleftrightarrow-\ldots \ldots \ldots \ldots$ Left
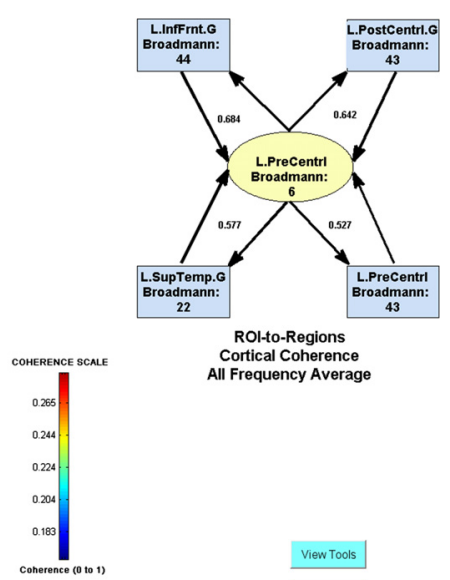

All Frequency Averag

Fig. 3 a MRI with MEG - CSI results imbedded. Color scale indicates Coherence level. Black circle indicates further analysis on the direction and locations of information flow. $\mathbf{b}$ Circle in center of diagram indicates location of black circle on MRI. The four strongest regions that are coherent with this location are displayed in the boxes; the numbers indicate the strength of the connection

patients could inform the choice of medication in this patient population, with increased coherence (i.e. increased excitability) being a promising biomarker for favorable responses to medications that limit excitatory transmission, such as benzodiazepines or antiseizure drugs. They have also used this technique to study the neural synchrony during eye gaze in patients with Autism Spectrum Disorder (ASD) [31]. Significantly higher coherence and synchronization in posterior brain regions (temporo-parietal-occipital) across all frequencies was evident in ASD patients, particularly within the low 0 to $15 \mathrm{~Hz}$ frequency range. Higher coherence in fronto-temporo-parietal regions was noted in controls. Significantly higher low frequency coherent activity in bilateral temporo-parieto-occipital cortical regions and higher gamma band coherence in right temporo-parieto-occipital brain regions during averted gaze was related to more severe symptomology as reported on the Autism Diagnostic Interview-Revised (ADI-R). This study suggests a pattern of aberrant connectivity that includes higher low frequency synchronization in posterior cortical regions, lack of long-range right hemispheric beta and gamma coherence, and decreased coherence in fronto-temporo-parietal regions necessary for orienting to shifts in eye gaze in ASD; a critical behavior essential for social communication. More research on imaging Biomarkers for ASD can provide a novel approach for bettering the lives of those with autism. Especially given that ASD is becoming more widespread, it is imperative that methods for diagnosis and prognosis are found.

\section{Conclusion}

The basis of functional networks in the brain is the neuronal oscillations. In this paper we reviewed the most common method used to measure and quantify the brain's synchronous oscillations which make up the network of brain connectivity (coherence). Coherence imaging has evolved from displaying the results in sensor space to the more advanced imaging techniques of displaying the results in sources space. There are several different software programs based on different source analysis signal processing techniques for converting the recorded MEG signals into their corresponding source 
locations in the brain. These techniques are currently being used to expand clinical knowledge in the field of neurology and neuropsychiatry.

We have highlighted some of the types of information that can be derived from imaging the connectivity of the brain networks across different pathologies. Detection of the synchronous activation of neurons can be used to determine the wellbeing or integrity of the functional connectivity in the human brain. In the future a combined anatomical, functional and effective connectivity mapping will become the mainstay of the clinicians for assessing and diagnosing normal and abnormal brain networks. Using a combined approach to understanding the brain networks will provide a more enduring multimodal biomarker than using one type of connectivity or network analysis alone. Overlaying the functional connectivity maps onto the structural connectivity images and then using direction of information flow derived from effective connectivity will provide an all-inclusive understanding of how the brain functions. These techniques will not only provide promising multimodal biomarkers of diseases but also help to provide individualized treatment therapies based on pre and post treatment brain connectivity imaging. With the ever evolving field of signal processing, computers and mathematics, we expect to see more sophisticated and powerful analytical neuroimaging methods developed and applied to functional neuroimaging data.

\section{Abbreviations}

EEG: Electroencephalography; MEG: Magnetoencephalography; fMRI: Functional Magnetic Resonance imaging; PET: Positron Emission Tomography; FFT: Fast Fourier transform; DICS: Dynamic imaging of coherent sources; M1: Primary motor cortex; MEG-CSI: MEG Coherence source imaging; MR-FOCUSS: Multi Resolution- FOCal Underdetermined System Solver; DMN: Default mode network; PCC: Posterior cingulate cortex; MPFC: Medial pre frontal cortex; ASD: Autism Spectrum Disorder; ADI-R: Autism Diagnostic Interview-Revised.

\section{Competing interest}

The author declares that she has no competing interests.

\section{Author's contribution}

SB carried out the literature reviews, wrote this review article and approved the final manuscript.

\section{Author's information}

SB is a Medical Physicist in the Neuromagnetism Lab, Department of Neurology at Henry Ford Hospital, where she performs functional brain imaging with a whole head Neuromagnetometer before patients undergo brain surgery.

Received: 22 October 2015 Accepted: 31 December 2015

Published online: 17 January 2016

\section{References}

1. Adler G, Brassen S, Jajcevic A. EEG coherence in Alzheimer's dementia. J Neural Transm. 2003;110:1051-8.

2. Başar $E$, Güntekin B. A review of brain oscillations in cognitive disorders and the role of neurotransmitters. Brain Res. 2008;1235:172-93.

3. Belardinelli P, Ciancetta L, Staudt M, Pizzella V, Londei A, Birbaumer N, et al. Cerebro-muscular and cerebro-cerebral coherence in patients with pre- and perinatally acquired unilateral brain lesions. Neuroimage. 2007;37(4):1301-14.

4. Boutros NN, Galloway MP, Ghosh S, Gjini K, Bowyer SM. Abnormal coherence imaging in panic disorder: a magnetoencephalography investigation. Neuroreport. 2013;24(9):487-91.

5. Bowyer S, Gjini K, Zhu X, Kim L, Moran J, Rizvi S, et al. Potential Biomarkers of Schizophrenia from MEG Resting-State Functional Connectivity Networks: Preliminary Data. J Behav Brain Sci. 2015;5:1-11. doi:10.4236/jbbs.2015.51001.

6. Brazier MA. Spread of seizure discharges in epilepsy: anatomical and electrophysiological considerations. Exp Neurol. 1972;36(2):263-72.

7. Bressler SL, Kelso JA. Cortical coordination dynamics and cognition. Trends Cogn Sci. 2001;5(1):26-36.

8. Cabral J, Kringelbach ML, Deco G. Exploring the network dynamics underlying brain activity during rest. Prog Neurobiol. 2014;114:102-31.

9. Chana G, Bousman CA, Money TT, Gibbons A, Gillett P, Dean B, et al. Biomarker investigations related to pathophysiological pathways in schizophrenia and psychosis. Front Cell Neurosci. 2013;7(95):1-18.

10. Classen J, Gerloff C, Honda M, Hallett M. Integrative visuomotor behavior is associated with interregionally coherent oscillations in the human brain. J Neurophysiol. 1998;79(3):1567-73.

11. de Pasquale F, Penna SD, Snyder AZ, Lewis C, Mantini D, Marzetti L, et al. Temporal Dynamics of Spontaneous MEG Activity in Brian Networks. Proc Natl Acad Sci U S A. 2010;107(13):6040-5. 
12. Elisevich K, Shukla N, Moran JE, Smith BJ, Schultz L, Mason KM, et al. An Assessment of MEG Coherence Imaging in the Study of Temporal Lobe Epilepsy. Epilepsia. 2011a; 52 (6):1110-9

13. Englot DJ, Hinkley LB, Kort NS, Imber BS, Mizuiri D, Honma SM, et al. Global and regional functional connectivity maps of neural oscillations in focal epilepsy. Brain Res. 2015;138(8):2249-62.

14. French CC, Beaumont JG. A critical review of EEG coherence studies of hemisphere function. Int J Psychophysiol. 1984;1(3):241-54

15. Friston KJ, Frith CD, Liddle PF, Frackowiak RS. Functional connectivity: the principal-component analysis of large (PET) data sets. J Cereb Blood Flow Metab. 1993;13:5-14.

16. Georgopoulos APKE, Leuthold AC, Lewis SM, Lynch JK, Alonso AA, Aslam Z, et al. Synchronous neural interactions assessed by magnetoencephalography: a functional biomarker for brain disorders. J Neural Eng. 2007;4(4):349-55.

17. Gerloff C, Richard J, Hadley J, Schulman AE, Honda M, Hallett M. Functional coupling and regional activation of human cortical motor areas during simple, internally paced and externally paced Wnger movements. Brain. 1998;121:1513-31.

18. Gotman J. Interhemispheric relations during bilateral spike-and-wave activity. Epilepsia. 1981;22:453-66.

19. Greenblatt RE, Pflieger ME, Ossadtchi AE. Connectivity measures applied to human brain electrophysiological data. J Neurosci Methods. 2012;2007(1):1-16.

20. Gross J, Kujala J, Hämäläinen M, Timmermann L, Schnitzler A, Salmelin R. Dynamic imaging of coherenc sources: Studying neural interactions in the human brain. Proc Natl Acad Sci U S A. 2001;98(2):694-9.

21. Gross J, Schmitz F, Schnitzler I, Kessler K, Shapiro K, Hommel B, et al. Modulation of long-range neural synchrony reflects temporal limitations of visual attention in humans. Proc Natl Acad Sci U S A. 2004;101(35):13050-5.

22. Gross J, Timmermann L, Kujala J, Dirks M, Schmitz F, Salmelin R, et al. The neural basis of intermittent motor control in humans. Proc Natl Acad Sci U S A. 2002;99(4):2299-302.

23. Guggisberg AG, Honma SM, Findlay AM, Dalal SS, Kirsch HE, Berger MS, et al. Mapping functional connectivity in patients with brain lesions. Ann Neurol. 2007;63:193-203.

24. Haenschel C, Linden D. Exploring intermediate phenotypes with eeg: working memory dysfunction in schizophrenia. Behav Brain Res. 2011;216:481-95.

25. Hamalainen M, Hari R, IImoniemi J, Knuutila J, Lounamaa O. Magnetoencephalography-theory, instrumentation and applications to noninvasive studies of the working human brain. Rev Mod Phys. 1993;65(2):413-97.

26. Hinkley LB, Vinogradov S, Guggisberg AG, Fisher M, Findlay AM, Nagarajan SS. Clinical symptoms and alpha band resting-state functional connectivity imaging in patients with schizophrenia: implications for novel approaches to treatment. Biol Psychiatry. 2011;70(12):1134-42.

27. Hoechstetter K, Bornfleth H, Weckesser D, Ille N, Berg P, Scherg M. BESA source coherence: a new method to study cortical oscillatory coupling. Brain Topogr. 2004a;16(4):233-8.

28. Horwitz B. The elusive concept of brain connectivity. Neuroimage. 2003;19:466-70.

29. Kim JS, Shin KS, Jung WH, Kim SN, Kwon JS, Chung CK. Power Spectral Aspects of the Default Mode Network in Schizophrenia: An MEG Study. BMC Neuroscience. 2014; 15(104). doi:10.1186/1471-2202-15-104

30. Lachaux JP, Rodriguez E, Martinerie J, Varela FJ. Measuring phase synchrony in brain signals. Hum Brain Mapp. 1999:8:194-208.

31. Lajiness-O'Neill R, Richard AE, Moran JE, Olszewski A, Pawluk L, Jacobson D, et al. Neural synchrony examined with magnetoencephalography (MEG) during eye gaze processing in autism spectrum disorders: preliminary findings. J Neurodev Disord. 2014;6(1):15.

32. Le Bihan D, Mangin JF, et al. Diffusion tensor imaging: concepts and applications. J Magn Reson Imaging 2001;13(4):534-46.

33. Lehmann D, Faber PL, Gianotti LR, Kochi K, Pascual-Marqui RD. Coherence and phase locking in the scalp EEG and between LORETA model sources, and microstates as putative mechanisms of brain temporo-spatial functional organization. J Physiol Paris. 2006;99(1):29-36.

34. Llinas RR. The Intrinsic electrophysiological properties of mammalian neurons: A new insight into CNS function. Science. 1988;242(4886):1654-64.

35. Llinas RR, Grace AA, Yarom Y. In vitro neurons in mammalian cortical layer 4 exhibit intrinsic oscillatory activity in the 10- to 50-Hz frequency range. Proc Natl Acad Sci U S A. 1991;88(3):897-901.

36. Locatelli T, Cursi M, Liberati D, Franceschi M, Comi G. EEG coherence in Alzheimers disease. Electroencephalogr Clin Neurophys. 1998;106:229-37.

37. Manganotti P, Gerloff C, Toro C, Katsuta H, Sadato N, Zhuang P, et al. Task-related coherence and taskrelated spectral power changes during sequential Wnger movements. Electroencephalogr Clin Neurophysiol. 1998;109(1):50-62.

38. Ogawa S, Lee TM, Kay AR, Tank DW. Brain magnetic resonance imaging with contrast dependent on blood oxygenation. Proc Natl Acad Sci U S A. 1990;87:9868-72.

39. Özerdem A, Güntekin B, Atagün M, Turp B, Başar E. Reduced long distance gamma (28-48 Hz) coherence in euthymic patients with bipolar disorder. J Affect Disord. 2011;132:325-32.

40. Sakkalis $\mathrm{V}$. Review of advanced techniques for the estimation of brain connectivity measured with EEG/MEG. Comput Biol Med. 2011;41:1110-7.

41. Schnitzler A, Gross J. Normal and pathalogical oscillatory communication in the brian. Nat Rev Neurosci. 2005:6(4):285-96.

42. Schoffelen JM, Gross J. Source connectivity analysis with MEG and EEG. Hum Brain Mapp. 2009;30(6):1857-65.

43. Song J, Tucker DM, Gilbert T, Hou J, Mattson C, Luu P, et al. Methods for examining electrophysiological coherence in epileptic networks. Front Neurol. 2013;4:55.

44. Stephan KE, Friston KJ, Frith C. Dysconnection in schizophrenia: From abnormal synaptic plasticity to failures of self-monitoring. Schizophr Bull. 2009;35:509-27.

45. Towle VL, Hunter JD, Edgar JC, Chkhenkeli SA, Castelle MC, Frim DM, et al. Frequency Domain Analysis of Human Subdural Recordings. J Clin Neurophysiology. 2007;24(2):205-13.

46. Uhlhaas PJ, Roux F, Rodriguez E, Rotarska-Jagiela A, Singer W. Neural synchrony and the development of cortical networks. Trends Cogn Sci. 2009;14(2):72-80. 
47. Uhlhaas PJ, Singer W. Abnormal neural oscillations and synchrony in schizophrenia. Nat Rev Neurosci. 2010;11(2):100-13.

48. Wang XJ. Neurophysiological and computational principles of cortical rhythms in cognition. Physiol Rev. 2010:90:1195-268.

49. Wedeena VJ, Wanga RP, Schmahmannb JD, Bennera T, Tsengc WYI, Daia G, et al. Diffusion spectrum magnetic resonance imaging (DSI) tractography of crossing fibers. Neuroimage. 2008;41(4):1267-77.

50. Yeragani VK, Cashmere D, Miewald J, Tancer M, Keshavan MS. Decreased coherence in higher frequency ranges (beta and gamma) between central and frontal EEG in patients with schizophrenia: a preliminary report. Psychiatry Res. 2006;141:53-60.

Submit your next manuscript to BioMed Central and we will help you at every step:

- We accept pre-submission inquiries

- Our selector tool helps you to find the most relevant journal

- We provide round the clock customer support

- Convenient online submission

- Thorough peer review

- Inclusion in PubMed and all major indexing services

- Maximum visibility for your research

Submit your manuscript at www.biomedcentral.com/submit 